
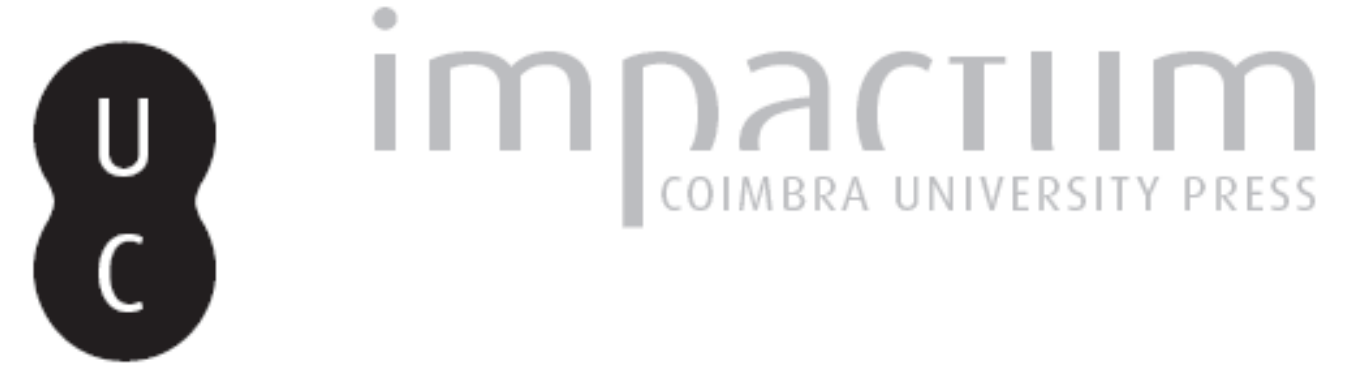

\title{
[Recensão a] Sylvain Delcomminette, Le Philèbe de Platon. Introduction à l'agathologie platonicienne
}

\author{
Autor(es): $\quad$ Pinchard, Alexis
}

Publicado por: Imprensa da Universidade de Coimbra

URL persistente:

URI:http://hdl.handle.net/10316.2/42196

DOI:

DOI:https://doi.org/10.14195/2183-4105_10_4

Accessed : $\quad$ 26-Apr-2023 13:36:36

A navegação consulta e descarregamento dos títulos inseridos nas Bibliotecas Digitais UC Digitalis, UC Pombalina e UC Impactum, pressupõem a aceitação plena e sem reservas dos Termos e Condições de Uso destas Bibliotecas Digitais, disponíveis em https://digitalis.uc.pt/pt-pt/termos.

Conforme exposto nos referidos Termos e Condições de Uso, o descarregamento de títulos de acesso restrito requer uma licença válida de autorização devendo o utilizador aceder ao(s) documento(s) a partir de um endereço de IP da instituição detentora da supramencionada licença.

Ao utilizador é apenas permitido o descarregamento para uso pessoal, pelo que o emprego do(s) título(s) descarregado(s) para outro fim, designadamente comercial, carece de autorização do respetivo autor ou editor da obra.

Na medida em que todas as obras da UC Digitalis se encontram protegidas pelo Código do Direito de Autor e Direitos Conexos e demais legislação aplicável, toda a cópia, parcial ou total, deste documento, nos casos em que é legalmente admitida, deverá conter ou fazer-se acompanhar por este aviso.

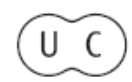



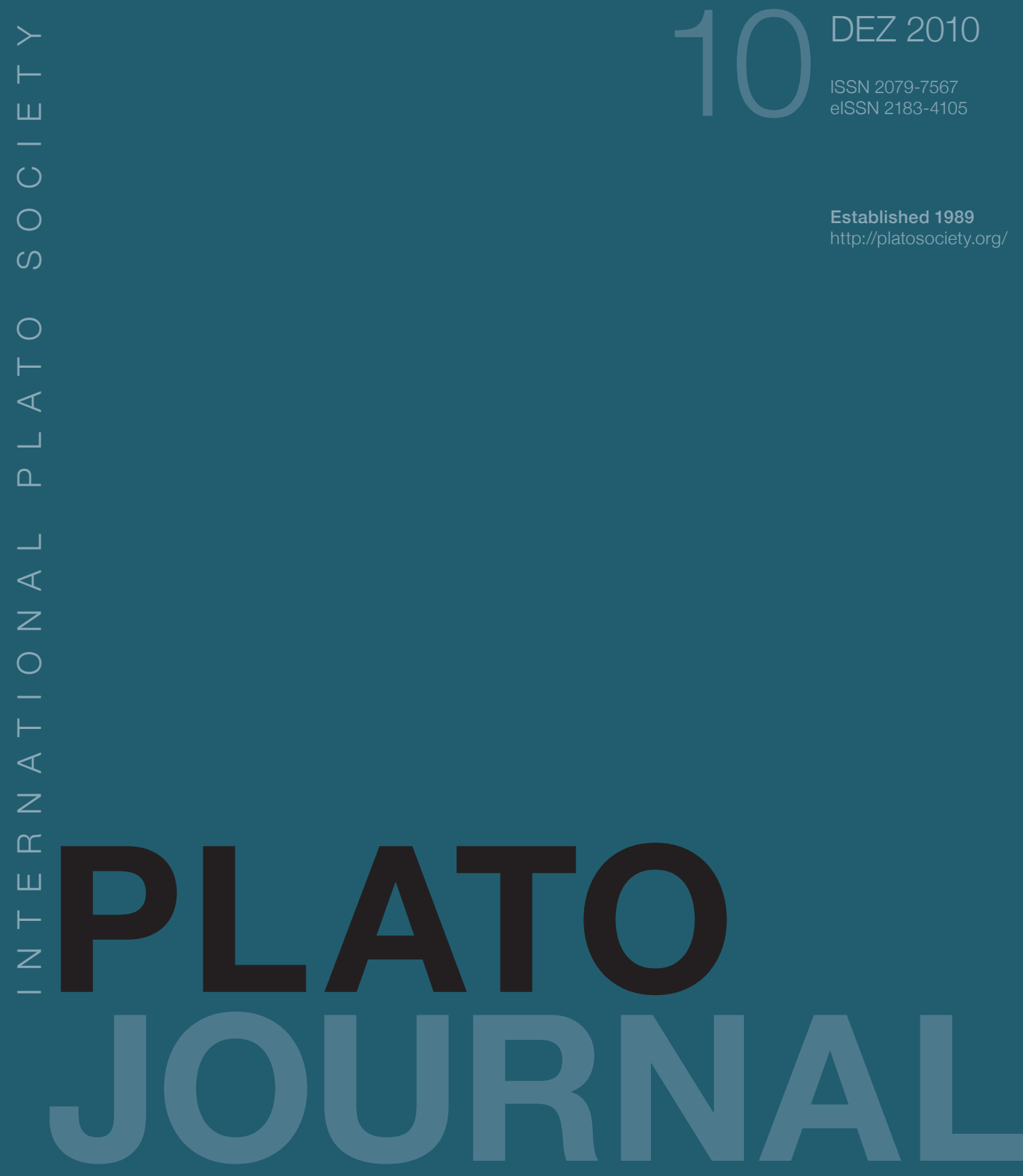

Société Platonicienne Internationale

Associazione Internazionale dei Platonisti

Sociedad Internacional

de Platonistas

Internationale

Platon-Gesellschaft 
PINCHARD, Alexis, « Compte rendu de S. Delcomminette, Le Philèbe de Platon, Introduction à l'agathologie platonicienne, Boston-Leiden, Brill, 2006 »

Sylvain Delcomminette, Le Philèbe de Platon. Introduction à l'agathologie platonicienne, Brill, 2006, Boston-Leiden ; bibliographie, index nominum et index locorum, $680 \mathrm{p}$.

L'ouvrage de Sylvain Delcomminette (abrégé ici par la suite «D.»), directement issu de sa thèse de doctorat, nous propose une interprétation d'ensemble de la pensée platonicienne à travers une lecture serrée du Philèbe, et cette visée systématique, quelque audacieuse qu'elle puisse paraître aux yeux de ceux qui voudraient discerner les méandres d'une histoire dans le l'œuvre multiforme de Platon, n'est pas le moindre de ses mérites. Même si l'on peut ne pas toujours en partager les conclusions, les analyses de Delcomminette déterminent les options conceptuelles à disposition du lecteur, pour la compréhension de la lettre du Philèbe comme pour des questions aussi générales que la nature de la dialectique ou le statut ontologique des Formes intelligibles chez Platon, avec une puissance et une précision qui mérite toute la gratitude des philosophes de métier et de cœur.

La thèse qui parcourt l'ouvrage s'inscrit d'emblée en rupture avec le néoplatonisme et ses émules modernes : il y a une science du bien en soi dans les écrits de Platon, pas plus mystique que la science des autres Idées quoiqu'elle en constitue l'ultime couronnement, et c'est le Philèbe, loin de se cantonner à la vie bonne, qui l'expose effectivement. Alors que l'allégorie solaire de la République se contentait d'affirmer la possibilité d'une telle science et en déterminait le rôle par rapport aux autres savoirs conformément à la fonction principielle du bien, le Philèbe prend en charge l'essence même du bien, qui seule rend pleinement raison de sa fonction. Le Philébe, en s'élevant graduellement de l'analyse du bien humain à celle du bien absolu, laquelle ne sera ni une hénologie, ni une ontologie, ni une éthique abstraite, confirme que l'essence du bien n'est étrangère par nature ni au logos oral ni au logos écrit tel que Platon le pratique. Il faut entendre ici « science » en un sens proprement platonicien : à la fois application continue de la méthode dialectique et résultat de cette méthode, c'est-à-dire saisie exacte de la Forme intelligible par la partie rationnelle de l'âme. Évidemment, celui qui se contentera de répéter, comme quelque incantation magique destinée à apaiser l'angoisse née de l'ignorance reconnue, les trois mots « Mesure », « Beauté », « Vérité » écrits à la fin du Dialogue ne possédera pas la moindre science. Pour

PLATO, The electronic Journal of the International Plato Society, n 10, 2010.

http://gramata.univ-paris1.fr/Plato/article96.html

(c) All rights of reproduction of any form reserved. 
accéder à « l'agathologie platonicienne » que nous promet le titre du livre, il faut effectuer personnellement le cheminement que ces mots récapitulent. Isolés, ils ne sont que des coquilles vides. La leçon platonicienne sur le bien demeurera inaudible pour le lecteur passif, au mieux une légende dorée, au pire une anecdote douteuse. La systématisation de la pensée platonicienne autour d'une doctrine du bien, dépassant les divisions scolaires entre éthique, cosmologie, esthétique et épistémologie pour les reconduire à quelque unité originaire, ne peut donc s'effectuer qu'au prix d'une reconstruction systématique du Philèbe lui-même. Et en ce qu'elle nous demande ainsi de faire ouvre de philosophie à notre tour, cette systématicité n'a rien d'une borne imposée a priori à la pensée. L'agathologie est un élan, non un cadre. C'est pourquoi la méthode herméneutique de D. consiste à ré-effectuer pour soi et de l'intérieur les étapes logiques du texte en écartant tout témoignage indirect sur l'enseignement de Platon, jusqu'à abolir la différence entre philosophie et histoire de la philosophie. Pour cela, il faut évidemment postuler la parfaite cohérence du Dialogue, postulat qui sera validé a posteriori par le succès de la démarche entreprise en son nom.

La Première Partie de l'ouvrage étudie à la fois l'élaboration progressive du problème du rapport entre le plaisir et le bien ainsi que la méthode proposée pour le résoudre - la dialectique, tandis que la Deuxième Partie se consacre à l'application de cette méthode en tant qu'elle produit les divisions spécifiques des plaisirs et des sciences. Enfin, la troisième Partie en vient à l'Idée du bien ellemême. Dans ce compte rendu, nous suivrons l'ordre des parties du livre de D., en développant plus spécialement la première parce que les interprétations qui y sont proposées sont la condition de l'originalité de l'ensemble. En outre, nous suggérerons au fil des arguments quelque remarques critiques destinées à montrer que si D. repère et élabore bien tous les problèmes importants du Philèbe, il ne les résout pourtant pas toujours entièrement - ce qui d'ailleurs va dans le sens de la perpétuation de l'activité dialectique qu'il appelle de ses vœux.

D. commence donc par repréciser les thèses des interlocuteurs en présence, reconnaissant que Platon les présente à dessein avec un certain vague, du moins au début : la discussion émerge sur fond d'indéterminé (apeiron) et elle est là pour obliger chacun à se déterminer. Philèbe, tel du moins que le comprend Socrate, soutient que le plaisir est bon par essence, c'est-à-dire par cela seul qu'il est plaisir, en sorte que le plaisir est une espèce du bien, tandis que Protarque,

PLATO, The electronic Journal of the International Plato Society, n 10, 2010.

http://gramata.univ-paris1.fr/Plato/article96.html

(c) All rights of reproduction of any form reserved. 
essayant de traduire la manière dont Philèbe mène sa vie dans l'espace du logos, rapproche encore davantage les deux termes en soutenant l'identité pure et simple du plaisir et du bien, sans concevoir que le bien puisse être un prédicat applicable à d'autres sujets. Bref l'un soutient que tout le plaisir est bon, tandis que l'autre voudrait que seul le plaisir soit bon. Quant à Socrate, il ne nie pas que certaines espèces de plaisirs puissent être bonnes, mais il refuse tant la prédication essentielle du bien à l'égard du plaisir et que l'identification du bien au plaisir comme il refusera l'identification pure et simple du bien aux activités intellectuelles, même si toutes, à des degrés divers, sont bonnes. L'identification immédiate du bien au plaisir constitue la thèse à réfuter en premier car elle interdit toute prédication du bien à d'autres sujets que le plaisir - essentielle ou accidentelle - , et donc toute mise en œuvre de la dialectique ; or Socrate fait du bien une différence spécifique au sein du plaisir. Néanmoins cette dernière position ne pourra être justifiée qu'en déterminant ce qu'est le bien en lui-même, ce qui nous mènera évidemment à l'Idée du bien. Le lecteur s'attendrait ici à une référence à la méthode proposée dans le Phédon (100 a3-7) qui veut que, pour répondre à toute question concernant les choses particulières, question de causalité ou de prédication («ceci est-il beau ? Pourquoi ceci est-il beau ?»), on commence par poser un logos idéel, qui peut être un logos définitionnel. En effet, pour savoir si une chose est belle, il faut tout d'abord définir la beauté. Néanmoins D. n'effectue pas ce rapprochement tentant.

Ainsi, face à Protarque qui ne veut pas reconnaître que les plaisirs peuvent différer les uns des autres sous le rapport de leur bonté en tant que plaisirs et non pas seulement en tant qu'effets de causes diverses, comme les couleurs different les unes des autres en tant que couleurs et non par leur seul support, Socrate argue tout d'abord du fait que même un homme dépourvu d'intelligence peut éprouver du plaisir ; mais cela ne constitue une espèce mauvaise du plaisir que pour celui qui a reconnu que la stupidité et l'ignorance sont des maux tandis que leur contraire sont des biens, et que donc le bien est originellement autre chose que le plaisir, ce qu'a d'emblée refusé Protarque. Que le plaisir garde son unité générique tout en se différenciant intrinsèquement, bref que les plaisirs soient à la fois semblables et dissemblables à d'autres plaisirs semble inconcevable à Protarque. Une progression de la discussion sur la base d'un accord mutuel semble donc impossible. Pour lever cet obstacle, il faut établir comment quelque chose en général, y compris le plaisir, quelque contradictoire que cela paraisse, peut être à la 
fois un et multiple. Cela implique deux ordres de problèmes, les premiers puérils, construits par la sophistiques mais facilement résolus par la dialectique en tant qu'elle distingue le sensible et l'intelligible, les seconds sérieux, surgissant justement avec l'opération du dialecticien sur les Formes intelligibles mais non résolus dans le Philèbe ; ce Dialogue qui se contente d'appliquer la dialectique sans en établir les conditions de possibilité, suppose la solution de ces problèmes sérieux telle qu'est élaborée dans le Théétète, le Sophiste et le Politique, et en même temps fournit le principe organisateur de toute l'œuvre platonicienne de la maturité. On obtient ainsi deux séries de problèmes analogiques $(2+3)$, point sur lequel l'interprétation de $\mathrm{D}$. se signale par son originalité et sa puissante cohérence. Le premier problème puéril, déjà posé et résolu dans le début du Parménide (129a-b), se pose lorsqu'on exhibe le mélange de l'un et du multiple effectué par les prédications de deux contraires — ou même simplement de deux qualités différentes - au sujet de la même chose sensible : dès qu'on déplace la multiplicité vers un autre plan ontologique en admettant l'existence des Formes séparées, cette multiplicité de prédicats se résout en une multiplicité de participation qui ne remet plus en cause l'unité du sujet. En outre, ce n'est pas ce type d'entrelacement entre l'un et le multiple qui est convoqué par la division du plaisir en ses espèces, puisque le plaisir demeure une notion générale, et non une chose particulière sensible. Le second problème puéril concerne la contradiction apparente entre l'unité du tout et la multiplicité des parties dans une même chose sensible: le dialecticien se contente d'interpréter cette situation en affirmant qu'une même chose sensible participe à l'Idée de l'un et à l'Idée du multiple, comme elle peut participer au semblable et au dissemblable ainsi que l'établit Socrate dans le Parménide. La solution du second problème puéril constitue donc un cas particulier de la solution du premier. De même, ce n'est pas non plus à ce type de rapport entre l'un et le multiple qu'auront affaire ceux qui veulent distinguer des plaisirs bons et des plaisirs mauvais, quoiqu'il fournisse une image dégradée de la synthèse d'un et de multiple opérée par le dialecticien : les espèces ne sont certes pas des composants du genre comme des parties au sein d'un agrégat sensible, mais des déterminations supplémentaires qui viennent se composer avec celles du genre, se tenant pourtant encore dans l'unité d'un même concept $(\log o s)$; la totalité des déterminations spécifiques constitue donc bien l'unité d'une multiplicité, mais cette totalité est logiquement antérieure à la

PLATO, The electronic Journal of the International Plato Society, n 10, 2010.

http://gramata.univ-paris1.fr/Plato/article96.html

(c) All rights of reproduction of any form reserved. 
multiplicité qu'elle renferme alors que, dans le sensible, le tout n'est jamais que la somme de ses parties.

Les trois problèmes sérieux, quant à eux, soulèvent tout d'abord une difficultés textuelle, tous les traducteurs - notamment, en France, Diès n'étant pas d'accord pour reconnaître trois problèmes dans le passage en question (15 b1-8) en raison de l'obscurité de ce qui est censé tenir lieu de second problème, à tel point que certains commentateurs proposent de corriger le texte transmis par les manuscrits pour éliminer la répétition du démonstratif $\tau \alpha \dot{v} \tau \alpha \varsigma .$.

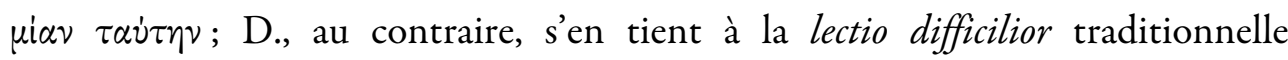
imprimée par Burnet, tranchant les incertitudes de la grammaire et de la philologie au nom de la cohérence de son interprétation. Le premier problème concerne l'existence des Idées en tant que conditions de possibilité de l'exercice de la dialectique, ainsi que l'a déjà reconnu la majorité des commentateurs. Ce problème, quoique ne faisant pas intervenir le multiple face à l'un, constitue le pivot qui nous fait basculer dans l'entrelacement de l'un et du multiple au sein de l'intelligible, alors que les problèmes puérils ne concernaient que le sensible, ou plutôt résultaient de la non-différenciation du sensible et de l'intelligible. Néanmoins il ne s'agit pas seulement de se demander si les Idées existent, mais, de manière critique et réflexive, de se demander les raisons qui peuvent légitimement amener à poser l'existence de telles unités. D. insiste ici sur l'intervention subjective du philosophe : les Idées ne sont en aucun cas des «éléments de la réalité », mais des «hypothèses faites par le philosophe» (p. 62). Par conséquent, croit pouvoir conclure $\mathrm{D}$. de manière assez surprenante, les raisons qui peuvent inciter à affirmer l'existence des Idées ne consistent pas nécessairement dans l'adéquation d'une telle affirmation avec la réalité (mais alors que devient la vérité d'une telle affirmation, vérité pourtant présente dans le texte du Philèbe avec l'adverbe $\dot{\alpha} \lambda \eta \theta \tilde{\omega} s$ ?). Il se pourrait, selon D., que ce soit plutôt l'exigence de la possibilité de la science qui oblige le philosophe à une telle hypothèse : pour qu'une science soit possible, il faut poser l'existence réelle des Idées. Cette interprétation sera reprise p. 595, où il est dit que les Formes sont quelque chose que l'âme « se donne » et que le problème n'est jamais de savoir s'il y a ou non au monde quelque chose de tel. Mais le lecteur demeure en droit de se demander si en effet une science est possible. Le seul désir de science ne suffit pas à en réaliser de manière certaine la possibilité, en sorte que le désir de science ne peut trancher en vérité la question de l'existence des Idées comme conditions

PLATO, The electronic Journal of the International Plato Society, n 10, 2010.

http://gramata.univ-paris1.fr/Plato/article96.html

(c) All rights of reproduction of any form reserved. 
de possibilité de la seule science digne de ce nom, la dialectique. Kant, qui imprègne le vocabulaire de $\mathrm{D}$. à plusieurs reprise, notamment dans la distinction entre « forme » et «matière » des Idées, ne nous a-t-il pas appris que les Idées nécessairement posées par la raison pour orienter les efforts de synthèse de l'entendement ne renvoient à aucune réalité objectivement connaissable, quelque tentantes que soient les apparences sur ce point? Que je doive penser quelque chose n'implique pas que cela doive exister : la nécessité qui se fait sentir dans ma pensée ne saurait contraindre les choses. D. ne semble ici pas faire la différence entre deux types d'hypothèses concernant la science, assimilant abusivement le premier au second: d'une part une hypothèse pour ainsi dire objective, de la forme «si la science est possible dans l'absolu », hypothèse qui n'étant pas assurée par elle-même, ne saurait non plus assurer ses conséquences, fussent-elles « les Idées existent »; d'autre part une hypothèse pour ainsi dire subjective, du type «si je veux construire une science », qui ne peut être démentie, et dont les conséquences pourraient se formuler de manière impérative : «alors je dois me donner l'existence des Idées ». L'hypothèse que nous appelons subjective n'a évidemment de sens que si l'hypothèse objective n'est plus une simple hypothèse mais une proposition nécessaire, par elle-même ou en vertu d'une autre proposition elle-même nécessaire. Autant Platon évoque, dans son œuvre (à la toute fin du Cratyle par exemple) le premier type d'hypothèse en en reconnaissant le caractère sinon incertain, du moins indémontrable a priori, autant il nous semble étranger au renversement de la hiérarchie entre prâxis et theôria implicitement contenu dans la connexion entre la seconde hypothèse et sa conséquence, renversement qui relève plutôt d'un certain idéalisme allemand, celui de Fichte par exemple, voire du marxisme. Qu'un projet psychique individuel de production du savoir puisse donner vérité à l'affirmation que les Idées existent risque de nous faire retomber dans l'homme-mesure de Protagoras. Si la volonté de D. d'éviter l'écueil du « monde intelligible » est louable, il nous semble glisser trop facilement, malgré ses dénis explicites (p. 281 entre autres), de l'évidente intériorité de l'intelligible à un rapport de dépendance entre l'Idée et le sujet désirant savoir. Ce n'est plus que la sincérité de l'engagement rationnel de l'individu en question, qui nous sauve d'un relativisme individualiste. En termes hegeliens, la certitude de soi invoquée par D. croit pouvoir se satisfaire d'ellemême et faire l'économie de l'épreuve de la vérité comme moment transcendant. Ce faisant, la différence entre Idée et concept s'efface, ou bien l'Idée est réduite à 
quelque foyer imaginaire symbolisant le pouvoir de synthèse mis en œuvre par l'entendement dans la dialectique. D'ailleurs, D. soulève ces difficultés à l'occasion de sa lecture du second problème sérieux, mais sans les résoudre, estimant que cela a été suffisamment fait dans le Sophiste et que ce n'est pas l'objet de son ouvrage.

Le second problème, en effet, concerne, selon D., la cohabitation de l'un et du multiple au sein du concept de chaque espèce. D. appuie cette interprétation sur la critique par Aristote des définitions platoniciennes produites par dichotomie (Métaphysique Z, 12, 1037 b13-14 ; Partie des Animaux I, 2-4), prenant au passage quelques liberté avec le principe méthodologique - proclamé plus haut - d'une lecture strictement interne du Philèbe. Cette critique aristotélicienne met en évidence les difficultés rencontrées par les platoniciens pour donner de l'unité à leurs longues définitions en raisons de leur postulat selon lequel les universaux, y compris ceux qui servent à introduire une différence au sein du genre, ont tous une existence séparée tant à l'égard du sensible qu'à l'égard les uns des autres. Platon lui-même aurait donc pressenti ce danger. Par conséquent, dans le second problème sérieux de l'un et du multiple mentionné dans le Philèbe, il ne s'agit pas immédiatement de la pluralité des espèces présentes dans un unique genre, mais bien d'une pluralité idéale de déterminations présente dans toute Forme intelligible, y compris spécifique, même si - D. suggère ce point plus qu'il ne le développe - une telle pluralité n'aurait pas lieu d'être en cas de non-pluralité des espèces : les multiples déterminations d'une espèce sont aussi, du moins en partie, ce qui la distingue d'autres espèces et ce qui fait donc la pluralité des espèces relevant du même genre. Davantage, ne peut-on affirmer que, en règle générale, plus les espèces ou sous-espèces sont riches en déterminations internes, plus elles sont nombreuses au sein du genre ? Dans le Philèbe, le genre n'est pas à la fois un et multiple, mais seulement un : il est le rapport sous lequel un terme tel que «le plaisir » ou «l'illimité » peut se montrer à nous comme un, tandis que les espèces sont les rapports sous lequel ce même terme peut se montrer multiple. Et c'est la dialectique, bientôt décrite à de partir de $16 \mathrm{c}$ précisément en ces termes, qui met en évidence le fait qu'une même réalité pensable peut à la fois être une et multiple pour celui qui veut construire une science, c'est-à-dire à la fois genre et espèces. En effet, les espèces au sein d'un même genre se différencient selon les diverses Idées auxquelles le genre peut être amené à participer ; or c'est la dialectique, comme on le sait depuis le Sophiste, qui 
détermine ces relations de participation horizontale. Ainsi D. procède à une relative extériorisation des espèces à l'égard du genre : ce dernier n'est jamais ni la simple somme des espèces ni la matrice à partir de laquelle procéderaient les espèces selon une hiérarchie ontologique objective comme en ont conçu les néoplatoniciens. Les espèces ne préexistent pas dans les genres, ni en acte ni en puissance. Assez étrangement, D. précise même que la relation entre genre et espèces doit « être interprétée comme une relation dialectique plutôt que comme une relation ontologique » (p. 67, note 34), comme si Platon, en faisant de la philosophie une « chasse aux êtres », n'avait pas rendu une telle alternative plus que difficile! Selon D., les espèces, telles de nouvelles Idées, sont véritablement constituées par l'opération du dialecticien qui fait surgir pour notre connaissance les diverses participations du genre à d'autres Idées qui le différencient de l'intérieur, de même que le dialecticien mettait aussi en évidence la participation d'une même chose sensible à diverses Idées pour sauver son unité malgré la multiplicité des prédications dont elle pouvait être le sujet. Pour ainsi dire, une Idée naît de la communion de plusieurs sous l'effet du logos dialectique. Ainsi D. en vient à douter très explicitement de l'indépendance des Idées à l'égard du dialecticien, reconnaissant qu'une telle dépendance heurterait de front l'affirmation réitérée par Platon de l'éternité des Idées. Et le problème est laissé en suspens. Certes, du même coup, D. peut donner sens à la mention de « génération » et du «trépas » en 15 b 3, au cœur de ce qu'il identifie comme le second problème sérieux de l'un et du multiple. Néanmoins la procession néoplatonicienne, en particulier celle de Proclus, systématiquement rejetée par D., aurait pu aussi produire le paradoxe d'une génération dans l'éternité sans confondre génération et construction logique, tandis que la conversion aurait donné sens au «trépas ». D. semble négliger le fait qu'une dynamique de l'intelligible n'est pas nécessairement subjective, et qu'elle peut bien être justement de l'intelligible et non pas seulement par rapport à l'intelligible. Voulant retirer tout aspect hiératique à l'intelligible platonicien, il est à craindre qu'au contraire, de la sorte, D. lui en rajoute, le rendant immobile à jamais. En tout cas, que la pluralité des déterminations au sein de l'unité d'un même concept spécifique soit difficile à concevoir, le comportement de Protarque le prouve : il voulait à tout prix faire de la diversité des plaisirs une expression de la diversité des causes de plaisirs, plaçant ainsi la multiplicité en dehors du plaisir lui-même tandis qu'il gardait le plaisir dans l'unité infrangible de son indentification au bien. $\mathrm{Au}$ 
contraire, Socrate va lui demander de penser la participation limitée du plaisir au bien, produisant ainsi une multiplicité intelligible $d u$ plaisir.

Enfin, le troisième problème sérieux de l'un et du multiple concerne la participation et aurait déjà reçu sa solution dans le Politique. La doctrine ontologique du mélange de limite et d'illimitation n'y apporte rien de plus. Après avoir évoqué les conditions de l'exercice de la dialectique, puis les processus de constitution d'un intelligible complexe qu'elle met en œuvre, Platon s'interroge à présent sur sa connexion avec la « réalité sensible » (p.74). On voit donc que ces trois problèmes sérieux sont suscités par la manière dont la dialectique traite l'entrelacement effectif de l'un et du multiple dans les prédications du langage de tous les jours, tandis que les problèmes puérils sont le résultat de son interprétation sophistique. En ce sens, dialectique et sophistique traitent de la même chose: l'intelligible n'est pas convoqué par la dialectique comme un domaine d'objets particulier, mais comme un principe d'explication. Le Philèbe développe une réflexion immanente aux logoi - logoi dont «le plaisir est bon » fournit l'exemple principal - et abandonne le problème de la correspondance entre les choses et leurs divers onomata ou entre un même onoma - un nom commun - et ses diverses références. Ni l'un ni le multiple que la dialectique tente d'ordonner ne sont à chercher du côté de la référence des noms, si bien que l'identification problématique de l'un à l'autre ne saurait s'y jouer. Tout est affaire de sens, si l'on s'en tient à la dichotomie de Frege : une fois intégrés dans énoncés prédicatifs, les noms, malgré leur unité, prennent plusieurs sens. Nous reconnaissons là la volonté de désontologiser le Dialogue propre à D., déjà aperçue précédemment. Peut-être eût-il été pertinent d'envisager que ce soit notre perception des êtres qui entrelace l'un et le multiple sous l'effet des logoi (cf. 15d: $\pi 0 \lambda \lambda \dot{\alpha} \dot{\nu} \pi \circ \lambda \dot{o} \gamma \omega \nu)$, mais non les logoi eux-mêmes. Cet entrelacement serait-il vécu comme un problème, une contradiction à dépasser, s'il ne visait en définitive l'être de ce dont on parle ? Le principe de contradiction n'est-il pas d'abord un principe ontologique? $\mathrm{Ou}$ alors on transforme les noms en une multitude êtres autonomes, mais alors on n'a fait que déplacer le problème sans en changer la nature. Le «pathos immortel » (15d) qui consiste à identifier l'un et le multiple et qui réclame le remède de la dialectique est un affect « en nous » en tant que sujets de la connaissance spéculant sur l'être, et non un affect dans les logoi. Vouloir ainsi procéder à une évacuation du « problème de la référence » (p. 82, note 57), afin que, délivré de toute confrontation au sensible - car la référence 
est nécessairement sensible ? - le philosophe puisse venir à bout de sa tâche par la seule dialectique, c'est peut-être risquer une abstraction que la Lettre VII récuserait : en vérité, d'un côté l'ousia n'est pas un logos, et d'un autre côté la Dialectique est capable d'induire un frottement mutuel entre onomata, logoi, et sensations, jusqu'à ce que jaillisse l'étincelle de l'intellection qui nous met directement en présence de l'ousia elle-même, ayant dépassé tout intermédiaire représentationnel (cf. 344b).

D. poursuit en montrant que le choix de l'attitude spéculative envers les logoi - sophistique ou dialectique - n'est pas étranger au thème du Dialogue : c'est notre rapport au plaisir qui la détermine. Ceux qui se laissent guider par le plaisir choisiront la sophistique, tandis que ceux qui choisissent la dialectique pour elle-même auront le plaisir pour récompense sans même l'avoir poursuivi. Ainsi il faut avoir déjà décidé de subordonner le plaisir dans la conduite de notre vie aux impératifs de l'intelligence - bref vivre philosophiquement - pour pouvoir déterminer dialectiquement - et donc de manière vraie et certaine - le contenu de la vie bonne, contenu qui justement accordera, parmi ses diverses composantes, la dernière place au plaisir et la première à l'intelligence, et encore en admettant seulement certains plaisirs en lui, bref montrera que la philosophie est le bon mode de vie. Le choix de la philosophie comme méthode pour la connaissance du bien suppose ce que doit démontrer la philosophie, à savoir que le plaisir n'est pas le Bien et qu'il n'est pas bon par essence. On est donc ramené à un cercle existentiel: seuls ceux qui ont choisi de vivre philosophiquement peuvent découvrir que seule la philosophie constitue la vie bonne. Il y a un saut entre l'opinion et la philosophie ; on ne peut se persuader rationnellement de la valeur de la philosophie depuis l'extérieur de la philosophie. Un hédoniste comme Philèbe est incurable, tandis que Protarque pourra tout au plus sentir son erreur, sans pouvoir en comprendre les raisons. Cette circularité a un revers positif: connaître la vie bonne au sens fort du terme, avec tout le mouvement démonstratif que cela implique, et la vivre ne constituent qu'une seule et même chose. Autrement dit, « le Philèbe n'est pas seulement consacré à la vie bonne, il est la vie bonne $\gg$ (p. 285).

Quelle est donc cette méthode de connaissance, la dialectique, dont la pratique même doit nous rendre heureux, même si le résultat se dérobe ? D. choisit de séparer radicalement le problème des éventuelles sources pythagoriciennes du fameux passage décrivant la dialectique comme «méthode 
divine » (16 c-17 a) et le problème de son interprétation. La référence mythologique prestigieuse aux Ancêtres et à quelque Prométhée envoyé des dieux n'est lue que dans la perspective de sa fonction argumentative au sein du texte platonicien : il s'agit de faire admettre dogmatiquement à Protarque des principes qui ne pourront être justifiés que de l'intérieur de la pratique dialectique, une fois que celle-ci sera appliquée, puisque une science suprême - c'est le cas de la dialectique - ne saurait tenir ses principes d'ailleurs que d'elle-même. Une premier diallèle se trouve ainsi brisé : il faut une certaine ontologie - celle qui pose en tout être un mélange de peras et d'apeiria - pour rendre possible la dialectique, mais c'est l'exercice même de la dialectique, sur les notions de peras et d'apeiria, avec ses divisions et ses rassemblements (à partir de 23 e3-6), qui permettra de pleinement comprendre cette ontologie et d'en faire une science. Mais se trouve également évité le premier cercle existentiel signalé plus haut, à savoir que seule une conception correcte du bien, subordonnant le plaisir, permet de choisir la méthode à utiliser justement pour déterminer cette conception. Enfin, la dialectique constitue la part enseignable de toute technique, y compris cette la tekhnè que constitue la vie bonne. Ainsi, en décrivant la méthode dialectique, le Philèbe rend concrètement possible la conduite d'une vie bonne, et ne se contente pas de nous dépeindre un idéal abstrait, se tenant dans un lointain culpabilisant. Encore une fois les questions de méthode et les questions de contenu, au sein du Philèbe, montrent leur convergence, ou plutôt leur commun enracinement dans la question du bien. Et cette méthode dialectique, telle qu'elle est exposée dans le Philèbe, n'implique aucune évolution de fond dans la pensée de Platon par rapport aux Dialogues antérieurs; tout juste s'attelle-t-elle à des problèmes nouveaux, à savoir un énoncé prédicatif - «le plaisir est bon 》 - et non la relation entre un nom et sa définition, comme c'était encore le cas dans le Sophiste et le Politique. De fait, lorsqu'il aborde le détail du texte, cohérent avec lui-même, D. continue à minimiser l'impact ontologique du texte (certes il ne le nie pas formellement puisqu'il a admis un diallèle entre dialectique et ontologie ; mais reste à savoir où peut encore se cacher l'ontologie si l'on admet les prémisses

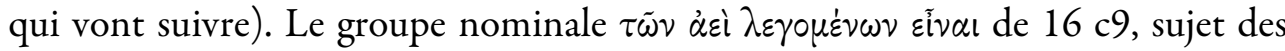
participes ő้ $\tau \omega \nu$ et $\dot{\varepsilon} \chi o \dot{\nu} \tau \omega \nu$ au sein des deux génitifs absolus coordonnées par $\mu \dot{\varepsilon} \nu . .$.

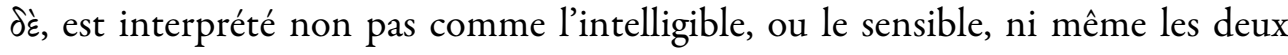
ensemble, qu'on mette du peras et de l'apeiria dans chacun des objets de ces domaines ou qu'on réserve le peras pour l'intelligible et l'apeiria pour le sensible 
— interprétations proposées jusqu’à présent —, mais comme les «termes généraux » intégrés dans des logoi à titre de sujet (« le plaisir ») et pris sous le rapport de leur sens, non de leur référence (c'est seulement dans le sens que l'intégration du terme général à un énoncé prédicatif produit du multiple tout supposant l'unité : le sens du mot «plaisir» semble changer lorsque l'on dit «certains plaisir sont bons» ou « certains plaisirs ne sont pas bons ». D. reproche aux interprètes antérieurs d'avoir fait intervenir trop tôt la division entre sensible et intelligible, alors que cette division est présentée comme la tâche à entreprendre une fois établi ce constat de l'entrelacement de l'un et du multiple au sein de ces termes généraux. Dans ces conditions, le participe passif $\lambda \varepsilon \gamma \sigma \mu \varepsilon \dot{\varepsilon} \nu \nu$ pèse plus lourd que l'infinitif हĩvaı qu'il introduit, lequel ne serait plus qu'à prendre comme copule pour un prédicat quelconque, donc impossible à expliciter. Et l'adverbe ảદi ne saurait renvoyer à quelque éternité positive. Mais nous pouvons aussitôt objecter à $\mathrm{D}$. qu'il néglige la préposition $\bar{\xi} \xi$ dans le début de

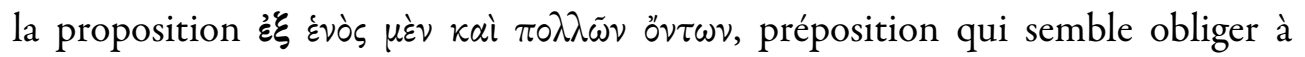
prendre le participe öv $\tau \omega \nu$ en un sens existentiel, et par là-même également l'infinitif عĩvaı auquel il est parallèle. À en croire D., « les choses qu'on dit à

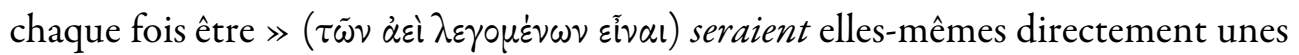
et multiples au lieu d'avoir leur être existentiel constitué à partir des éléments que sont l'un et le multiple. Il est évident que si D. admettait l'interprétation ontologique de ce passage, il devrait aussi admettre qu'il offre une solution aux trois problèmes sérieux énoncés plus haut et non pas seulement une description des opérations dialectique, ce que précisément il a refusé.

Ensuite D. établit une différence entre les deux moment du génitif absolus coordonnés par $\mu \dot{\varepsilon} v_{. .} \delta \dot{\varepsilon}$ : c'est à la dialectique de nous faire passer de l'affirmation selon laquelle les termes généraux du discours humains sont, quant à leur sens, à la fois uns et multiples, à celle selon laquelle ils contiennent en eux à la fois de la détermination - telle est la traduction de peras — dans la mesure où ils constitue des espèces distinctes les unes des autres de par leurs diverses caractéristiques internes, et de l'indétermination - telle est la traduction d'apeiron - dans la mesure où on prend comme des genres sans préciser les diverses espèces auxquelles auxquels ils peuvent donner lieu. Bref la dialectique est le processus par lequel l'intellect va isoler une polarité genre et une polarité espèces dans le terme général (le nom commun) qui sert de sujet à un logos quelconque, et ainsi mettre fin à la confusion dont il est l'occasion pour notre 


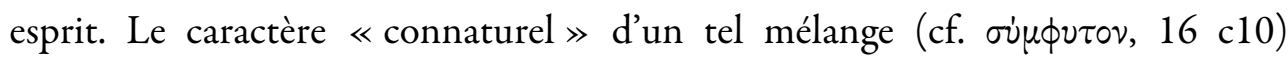
s'explique alors par le «pathos immortel » du langage humain (cf. $15 \mathrm{~d}$; est-ce donc encore un pathos agi par le langage en nous, ou subi par le langage lui-même comme semble le penser l'auteur ?), et non par une quelconque structure intime de l'être. Davantage, si les termes généraux peuvent ainsi contenir, imbriquées dans leur nature, la détermination et l'indétermination, c'est seulement parce que ceux-ci « résultent de la dialectique » (p. 100, note 91), en sorte qu'ils ne sauraient constituer des réalités en soi. L'apeiron, par exemple, n’appartient jamais à l'Idée, toujours parfaitement exacte et unie à elle-même, mais seulement à la pensée de l'Idée. Quand Platon, plus loin dans le Dialogue, nomme ơv $\tau \alpha$ (26 c5-6) le peras et l'apeiron, D. en refusera à nouveau toute ontologisation de ces principes sur la base de sa première analyse (p. 215: «Cela implique seulement que ces mots peuvent être référées à des entités qui peuvent être isolées l'une de l'autre d'une certaine manière »). N'eût-il pas été plus sage, au contraire, de corriger la première analyse au nom de la lettre insistante du texte platonicien ? En outre, que gagne-t-on à effectuer ce déplacement du couple peras/apeiron dans le processus dialectique, et donc dans l'âme ? Car on aboutit encore à des processus de pluralisation et d'unification progressives des concepts analogue à celui que les néoplatoniciens mettaient dans les Idées. Le schéma de progression de la connaissance reste le même, avec la même dynamique intérieure, sauf que l'on en a multiplié les instances, peut-être sans nécessité, ou au moins que l'on bascule vers une philosophie de la représentation. La dialectique, en tout cas, va devoir être comprise comme l'imposition progressive d'un peras à l'apeiron initial de notre pensée et non comme la découverte intuitive d'une hiérarchie préexistante dans l'être. Les Idées ne sont pas naturellement structurées en genre et en espèces, car elles ne sont pas du tout dans la nature des choses. Par exemple le plaisir pourrait être divisé sous un tout autre rapport que celui du bon et du mauvais. Cela n'empêchera pas la connaissance d'être vraie, dans la mesure où la vérité consiste dans l'exactitude intrinsèque de la pensée et non dans l'adéquation à un objet externe. L'auteur ne nous dit pas comment, dans ses conditions, il comprend le texte du Phèdre sur le dialecticien qui, tel un bon «boucher », respecte les « articulations naturelles » $(265 \mathrm{e} 2)$ au cours de ses divisions.

Ce constructivisme de la connaissance, qui n'évoque jamais l'hypothèse de la réminiscence, se fait sentir dès que l'on aborde la description des opérations dialectiques elles-mêmes, une fois passé le rappel de leurs conditions de possibilité. 
Le dialecticien doit commencer par poser de manière purement formelle une Idée du terme général qu'il examine, alors même que son contenu lui est encore inconnu : c'est au cours de l'exercice de la dialectique que cette position de l'Idée, initialement vide, se remplira, mais cet exercice n'est possible que parce que le dialecticien effectue tout d'abord une telle position. Il faut supposer quelque chose d'entièrement déterminé pour pouvoir entreprendre de le connaître, et cela ne peut ni ne doit être justifié. Il s'agirait là du principe « anhypothétique » dont parle la fin du livre VI de la République. Une telle Idée objective pour l'intellect l'aspect purement un de l'universel conceptuel. L'Idée d'un genre n'est que le rapport sous lequel le terme général est un, tandis que les Idées des espèces correspondent au rapport sous lequel il est multiple, mais en elles-mêmes les Idées ne contiennent aucune multiplicité. Davantage, la position de l'Idée a pour but de séparer l'unité et la multiplicité contenues dans le terme général étudié. D. insiste à plusieurs reprise sur ce caractère posé de l'Idée, et non découvert, notamment au nom du fait que Platon lui-même parle «d'hypothèse » au sujet des Formes. Ainsi toutes les Idées sont semblables quant à leur être-posé, qui constitue finalement leur «forme» d'Idée, par opposition à leurs contenus toujours particuliers. Il n'y a d'Idée que relativement à une perspective dialectique spéciale dont l'âme est la seule responsable. Mais cette subjectivation générale de l'intelligible oublie que l'hypothèse platonicienne consiste précisément à poser l'Idée comme ce qui ne dépend pas de ma pensée mais ce dont ma pensée dépend. D. semble vouloir fusionner le discours sur l'Idée et l'Idée elle-même, ou encore la modalité de l'hypothèse, impliquant effectivement l'acte contingent d'un sujet particulier, et le contenu de l'hypothèse, alors même qu'il reconnaît explicitement que Platon, y compris dans le Philèbe, revendique l'éternité et l'autonomie ontologique des Idées (p. 281). À vouloir toujours éviter l'écueil d'une mythologisation de l'invisible - exigence assurément légitime,$- \mathrm{D}$. réduit la valeur des Idées à leur valeur opératoire, se privant ainsi à nouveau de ce que l'on pourrait nommer, après Michel Foucault, la dimension «spirituelle» du platonisme : comment comprendre la transformation de l'âme sous l'impact de la connaissance de l'Idée si l'être de celle-ci se réduit à un être-posé-par-le-sujet ? Quel est ce sujet tout-puissant, qui se donne les thèses nécessaires à son entreprise scientifique ? Comment peut-il trouver un fondement en lui-même, le rendant définitivement propriétaire de son être et assuré de l'organisation de ses facultés cognitives, avant d'avoir réactualisé sa parenté avec l'être absolu ? En vérité l'âme 
se dit en plusieurs sens, et la meilleure preuve de la réalité des Formes réside dans le fait que leur rencontre peut changer l'âme radicalement, y compris en tant qu'elle est intellect (lui aussi est susceptible d'aliénation et le salut qui vient de lui suppose que lui-même soit d'abord sauvé), qu'elle le veuille ou non. L'automotricité de l'âme n'implique pas qu'elle soit intégralement cause d'ellemême. D. est bien péremptoire, même du point de vue de la lettre des Dialogues platoniciens tels que le Banquet (210e), lorsqu'il déclare, à propos de « l'intuition intellectuelle », que «personne ne semble [l'] avoir jamais expérimentée » (p. 281) en soi. En fait, si les Formes sont un instrument au service de l'intellect qui veut savoir, cet instrument change au moins autant celui qui s'en sert que ce à quoi il s'applique. Pour D., il deviendra difficile d'affirmer que la philosophie, en tant qu'effort pour penser les Idées, s'identifie, en l'homme, à la vie bonne, après avoir dénié à ces dernières, toute causalité « productive », laquelle sera réservée à l'intellect. Il devra limiter leur causalité à une causalité « logique », y compris pour l'Idée du bien, alors que le Phédon (100c-e) avait fait intervenir la causalité des Idées comme la seule causalité raisonnable, et qui plus est dans une discussion sur la génération et la corruption effectives des êtres, notamment l'âme.

Quoi qu'il en soit, la position initiale de l'Idée suscite aussi un apeiron corrélatif dans la pensée du dialecticien, qui n'est pas le nombre illimité des participants éventuels de cette Idée: avant la détermination dialectique du contenu de cette dernière, ces participants ne sauraient former pour nous des unités dénombrables. Cet apeiron, n'étant pas plus extensionnel qu'intensionnel, ne ferait que représenter pour nous la possibilité qu'a l'Idée d'être participée (p. 107), sans toutefois se confondre avec le soi-disant pouvoir objectif que cette Idée a de s'instancier, comme dans le néoplatonisme, et sans que cette Idée ne se confondre avec le concept qui seul inclut en lui-même la potentialité d'être prédiqué de plusieurs sujets (p. 103, sic). Cet apeiron concerne donc la multiplicité indéterminée des caractères qui distinguent les divers individus qui participent d'une même Idée, multiplicité dont il faut justement faire abstraction, c'est-à-dire laisser indéterminée, pour apercevoir la participation des individus en question à la même Idée. Mais comment surmonter cet apeiron strictement mental ? C'est ici qu'interviennent les processus de division du genre en espèces : il s'agit de produire une multiplicité limitée de concepts, caractérisée par un nombre à la fois cardinal et ordinal. Seule la série complète des espèces et leur ordre hiérarchique de succession permet de déterminer pleinement chacune 
d'elle. Le tout, ici, en tant qu'il est nombré, précède les parties. Cette série structurée, en tant qu'elle est pour et par la pensée, résulte d'une détermination de l'apeiron mental, mais cela n'implique pas que le contenu eidétique de chaque espèce ainsi obtenue donne à penser un tel mélange : sinon comment comprendre qu'il y ait une Idée générale et des Idées spécifiques de l'apeiron lui-même ? L’Idée n'a pas nécessairement les caractères de ce dont elle est l'Idée, et le fait purement formel d'être une espèce ne préjuge pas du contenu ontologique ainsi porté à la pensée. C'est aussi pourquoi la structure d'emboîtement des genres et des espèces produite par l'activité dialectique ne nous enseigne rien sur une quelconque hiérarchie ontologique des Idées. De même, le moment où le dialecticien doit relâcher sa pensée et la laisser aller dans l'apeiron dépend seulement de son projet cognitif du moment. Il n'y a pas d'espèce infime en soi : la dernière espèce à penser est celle après laquelle la division ne serait plus pertinente pour l'objet de la recherche.

L'analyse des exemples de pratique dialectique donnés par Socrate par la suite (17 e7-18 a6), qui illustrent respectivement le travail du professeur, à celui de l'élève et enfin à celui de l'inventeur, permet à $\mathrm{D}$. de confirmer la relative liberté avec laquelle le dialecticien choisit ses critères de division pour un terme général quelconque, ainsi que le nombre de fois où il doit répéter la procédure. Ainsi le son peut être divisé sous plusieurs rapports, quoique cette multiplicité ne soit pas non plus infinie et dépende finalement de ce qu'il est : ou bien selon l'aigu et le grave, ou bien selon la possibilité ou l'impossibilité d'être prononcé seuls. Dans le premier cas on obtient l'institution, par les fondateurs de la musique grecque, des différents types d'intervalles, déterminés à chaque fois par une fraction mathématique spécifique; dans le second cas la distinction des phonèmes en consonnes et voyelles. Ce n'est qu'au terme du travail dialectique de ces premiers Instituteurs que nous pouvons repérer les divers intervalles précisément comme tels : ceux-ci n'étaient pas naturellement données dans notre perception sensible. La sensation accompagnée d'un jugement déterminant, la perception à proprement parler, est le résultat de l'éducation. De même, dans la langue, le structuralisme nous a appris à reconnaître le caractère à la fois arbitraire et indivisible des systèmes phonétiques. Ainsi Socrate ne regroupe pas les divers phonèmes du grec parce qu'ils formeraient une unité naturelle, mais parce qu'ils ne peuvent être appris séparément les uns des autres. La notion de phonème acquiert son contenu du fait même de sa division en consonnes et voyelles, puis de

PLATO, The electronic Journal of the International Plato Society, n 10, 2010.

http://gramata.univ-paris1.fr/Plato/article96.html

(c) All rights of reproduction of any form reserved. 
la division des consonnes et des voyelles jusqu'au niveau des phonèmes individuels. Le son n'était pas naturellement phonétique avant cette entreprise dialectique, et même sur le plan phonétique d'autres divisions seraient possibles comme nous le montre aujourd'hui la comparaison des langues étrangères avec la nôtre. Reste à savoir si Platon avait conscience de ce que les phonèmes du grec n'étaient pas les phonèmes de toute langue possible! De manière générale, la structure mise au jour par le dialecticien n'appartient pas aux choses mais à la connaissance que nous en avons (cf. p. 154). Ce n'est pas un hasard si D., nous présentant un Platon où le sujet revendique une maîtrise souveraine sur tous ses actes cognitifs, rejoint ici le Descartes des Régles pour la direction de l'Esprit, lorsque celui-ci nous enjoint de distinguer les choses telles qu'elles sont en soi et telles qu'elles s'objectivent pour notre connaissance : certains regroupements et certaines mises en ordre peuvent s'avérer nécessaires pour la construction d'une connaissance objective, alors même que ce qu'on appelle communément le réel y est étranger (règle XII). Ainsi tout dialecticien doit répondre à la question « quelles divisions sont les bonnes dans le présent système de division ? », et non «quel système de division est le bon ? ( p. 155). Dans la présente enquête, le système de division des plaisirs s'articulera autour de la question de leur bonté. Le problème est qu'on ne sait pas encore ce qu'est le bien : ici, même le critère de division devra faire l'objet d'un examen dialectique.

Ce long excursus méthodologique a permis à Socrate de présenter la dialectique à Protarque, c'est-à-dire à quelqu'un qui ne peut pas encore la pratiquer en raison de sa conception erronée de la vie bonne: présentation extérieure donc. Symétriquement, Socrate va s'attacher désormais à définir le contenu de la vie bonne pour le même Protarque, c'est-à-dire pour quelqu'un qui ne peut pas pratiquer la seule activité capable de produire cette vie en l'homme, à savoir la dialectique : à nouveau présentation depuis l'extérieur. La seule manière de passer ainsi de l'extérieur à l'intérieur pour faire venir quelqu'un à la philosophie, bref la seule manière de briser le cercle au sein duquel le discours et l'expérience philosophique se conditionnent mutuellement, c'est de faire appel à quelque révélation divine comme Socrate l'a déjà fait avec les fameux «Anciens qui valaient mieux que nous ». Aussi est-ce à nouveau un souvenir inspiré qui va suggérer à Socrate l'existence d'un troisième terme, la vie mixte, capable de s'identifier à la vie bonne au-delà de la vie de pur plaisir et de la vie purement consacrée à la connaissance. Sera brisée par là l'identification pure et simple du 
plaisir au bien, position initiale de Protarque, en sorte qu'un examen de la participation du plaisir ou de certains plaisirs au bien, exprimée dans un logos prédicatif, pourra commencer. En analysant la vie de plaisir, Socrate va briser l'induction - menée par les hédonistes - qui mène de l'universalité universalité apparente de sa quête parmi les divers êtres vivants à son caractère digne de choix

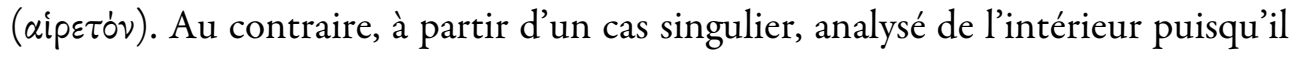
est question de la manière dont il faut vivre et que celui-là même qui mène l'analyse est vivant, Socrate va montrer que cette vie n'est pas digne de choix car celui qui la vit, n'ayant aucune conscience, n'a pas les moyens de choisir quoi que ce soit; or l'un des critères essentiels par lesquels on reconnait le bien est qu'il mérite d'être choisi pour lui-même. Le plaisir à l'état brut, sans la moindre trace d'intelligence, ne serait même pas vécu comme plaisir et donc ne pourrait être choisi pour ce qu'il est. Ainsi l'hédoniste lui-même, au nom de ses propres principes, se voit obligé de renoncer à l'identification du plaisir au bien. Socrate ne procède pas à une réfutation intellectualiste de l'hédonisme, laquelle n'aurait pu être acceptée par Protarque vu son anti-intellectualisme initial. Le cas de la vie de pure intelligence est différent : même si elle non plus ne se confond pas avec le bien, elle est néanmoins bonne par nature dans son ensemble, et non seulement elle est la condition de possibilité de tout plaisir mais elle produit d'elle-même effectivement certains plaisirs, lesquels figurent parmi les meilleurs. Les dieux, que nous devons et pouvons imiter, passent leur vie dans ces plaisirs-là, sans pour autant agir en étant motivés par eux - ils sont assez lucides pour savoir que le plaisir ne peut tenir lieu de fin dernière, c'est-à-dire de bien —, et sont dispensés des plaisirs qui résultent de la restauration d'une harmonie physique interne. La séparation de l'intelligence et du plaisir, condition de sa distinction à l'égard de la vie bonne, repose donc un effort d'abstraction artificiel, sans quoi on ne comprendrait pas que la vie bonne, à savoir la vie mixte, puisse finalement s'identifier à la vie philosophique.

De fait, après la détermination de la vie bonne comme vie mixte, toute la suite du Dialogue va tendre à montrer que c'est l'intelligence qui peut produire cette vie mixte en sorte que, dans un tel mélange, le plaisir n'obtient qu'une place subordonnée, à laquelle il ne peut même pas s'intégrer dans sa totalité. La question du rang axiologique respectif de l'intelligence et du plaisir au sein du mixte que constitue la vie bonne, très longuement débattue, n'est donc pas une annexe démesurée du Philébe permettant un simple exercice scolaire de la 
dialectique, mais en fonde l'ultime cohérence, rappelant l'unité originelle de la méthode théorique et de l'objet éthique auquel elle s'applique : la réponse à cette question va permettre de déterminer comme obtenir pour soi en réalité cette vie bonne et ainsi justifier la pratique de la philosophie qui parcourt le Dialogue d'un bout à l'autre. La simple réfutation de la thèse identifiant la vie bonne à la vie de pur plaisir, tout comme celle identifiant la vie bonne à la vie de pure intelligence, laissait encore ouverte la possibilité d'une vie bonne où l'intelligence, réduite à la conscience, aurait été ramené au rôle secondaire de condition de possibilité du plaisir. Les composants d'une vie mixte peuvent se hiérarchiser de diverses manières, et une seule d'entre elles est bonne. Autrement dit la fin du Dialogue peut se lire à la fois comme une progression dans l'analyse de la hiérarchie au sein composants de la vie bonne, ou comme une réinterprétation de la vie d'intelligence, laquelle se révèle peu à peu - du moins quand elle est choisie et donc concrètement expérimentée - nécessairement, mais exclusivement, suivie de plaisirs bons. La vie mixte va transformer les ingrédients qui y entrent: le plaisir, recevant détermination et proportion de la part de l'intelligence, ne devient bon qu'à condition d'y entrer, en sorte que sa valeur n'est qu'un effet de celle de l'intelligence. Diviser les plaisirs pour déterminer lesquels sont bon revient à les diviser selon la manière dont ils se mélangent à l'intelligence, qui seule permet d'en prendre conscience, ainsi qu'aux divers processus cognitifs dont cette dernière est le siège. Le dialecticien sait désormais sous quel angle il va devoir envisager les rapports de l'un et du multiple au sein de ce terme général qu'est le plaisir. Certes c'est parce que la vie mixte est mixte, douée qui plus est d'une certaine structure, qu'elle est bonne, et non parce que la seule intelligence y est présente : seule la participation à l'Idée de bien, en tant que « cause logique » (p. 197), peut légitimer que l'on juge quelque chose comme bon. La vie mixte constitue la demeure dans laquelle le dialecticien découvrira l'Idée du bien. Mais c'est l'intelligence, ne se réduisant pas quelque état passif contrairement au plaisir, qui est capable de faire advenir dans la réalité, au-delà d'elle-même, ce tout autosuffisant et harmonieux qu'est la vie participant à l'Idée de bien.

Pour justifier cette ultime identification de la vie mixte à la vie d'intelligence authentique, Socrate va devoir montrer que la vie humaine mixte bien hiérarchisée, subjectivement éligible entre toutes les formes de vie possibles et non pas seulement préférable dans certaines circonstances, correspond en réalité, sur un plan objectif, à un mélange de peras et d'apeiron constitué à l'image 
du monde dans sa totalité en tant que ce dernier est beau et mène une vie bienheureuse, espèce de mélange que seule une intelligence peut produire. En effet, il n'y a pas de mauvais mélange de peras et d'apeiron car seule une intelligence peut produire un tel mélange et ce que l'on sait de manière certaine être produite par elle - le monde - est assurément bel et bon. Malgré l'identification du plaisir à l'illimité, il faudra donc montrer que l'intelligence ne se confond pas avec la limite mais appartient à l'ordre de la cause qui la mélange à l'illimité, en sorte que la vie harmonisant limite et illimité, vie bonne s'il en fut, est bien produite par l'intelligence et que c'est l'intelligence, dans la vie mixte de plaisir et d'intelligence, qui tient le premier rôle. Voici donc revenir les catégories de la «méthode divine», mais cette fois-ci, semble-t-il, comme catégories ontologiques puisqu'il faut répartir en elles «parmi tout ce qui est maintenant dans l'univers » (23 c4-5, p. 213). Le sens de ses mots subit-t-il un changement au cours du Dialogue ? Non. Tout d'abord ces catégories n'existent pas en soi de toute éternité ; elles ne surgissent que quand le dialecticien veut classer les êtres en fonction de leur rapport à une intelligence productrice. Par exemple, l'apeiron n'est rien d'autre que ce qui échappe à l'intelligence productrice. L'intelligence est responsable non de la matérialité des choses, mais du fait que cette matérialité nous apparaît comme apeiron. Ensuite, avec cette division, il s'agit en vérité d'expliciter la solidarité du plan méthodologique et du plan éthique car cette classification exhaustive des êtres - propre à décrire la vie mixte puisque le plaisir par lui-même appartient au genre de l'illimité et que seule l'intelligence va pouvoir lui imposer les déterminations qui, du moins en partie, le rendent bon -, va à fois s'opérer selon la méthode de division et de rassemblement exposée en 16c, et produire, parmi ces quatre espèces, trois des concepts permettant explicitement de théoriser cette fameuse méthode dès $16 \mathrm{c}$ : le peras, l'apeiron, et le mixte. Il y a donc, à nouveau, une circularité: l'ontologie, née de l'application de la dialectique, est ce qui fournit, au-delà de toute révélation divine mythique, les concepts à l'œuvre dans la dialectique. Autrement dit: « ce que nous devons présupposer [pour pouvoir exercer la dialectique] n'est pas une ontologie extérieure, mais seulement que la dialectique peut s'appliquer à tout problème que nous rencontrons, dans la mesure où l'univers peut être décrit conformément aux catégories qu'elle met en œuvre (peras et apeiron)» (p. 284). Bref, «la dialectique ne se fonde sur rien d'autre qu'elle-même et la présupposition de sa propre possibilité » (p. 284). Au fond, le fait que la dialectique produise du mixte 
intérieur à l'intelligence en déterminant progressivement notre pensée des Idées à partir d'un état d'indétermination initiale nous garantit que la vie où l'on pratique philosophie, notamment pour déterminer ce qu'est le bien, sera bien une vie harmonisant, sur le plan ontologique, peras et apeiron à l'image de ce qui se fait à l'échelle du monde, lequel mène assurément une vie bonne. On valide ainsi ce qui a été sous-entendu par Socrate dès le début du Dialogue: apprendre à connaître la vie bonne et la vivre sont une seule et même chose. Il faudra donc admettre qu'il y a une Idée de l'apeiron, tout comme du peras, même si toute Idée, en tant que telle, est en elle-même parfaitement déterminée et que notre pensée de cette Idée, au terme du processus dialectique, est elle-même un mixte de peras et d'apeiron produit par notre intelligence à partir d'un état d'indétermination initial, à titre de cause. La « forme » idéelle qui appartient à toutes les Idées n'a rien à voir avec le «contenu» des Idées, et n'est même pas le genre qui rassemblerait les traits communs aux divers contenus des diverses Idées. De même, il y a des Idées de mixte, sans que les Idées ne soient elles-mêmes des mixte, ce qui confirme que le peras et l'apeiron supposés en 16c n'étaient en aucun cas des constituant de l'intelligible, mais des moments d'une procédure rationnelle. Certes certaines Idées, telles que la Beauté, semblent porter dans leur être-idée la marque même de leur contenu: ainsi Platon dit-il que la Beauté est non seulement belle, mais la plus belle de toute chose, mais cette « auto-prédication » n'est que le fait de leur contenu spécifique. Sinon la dialectique ne pourrait pas s'attaquer à son autre, à savoir le plaisir, produisant un concept déterminé de son impensabilité même, ainsi qu'elle le fait effectivement dans le Philèbe. Mais cette séparation entre la forme et le contenu des Idées ne risque-t-elle pas de faire éclater leur être, renouvelant, au moins de manière analogique, la séparation scolastique de l'existence et de l'essence, alors même Platon fait tous ses efforts pour les maintenir unies dans l'ousia? Quel sens donner, dans ces conditions, au

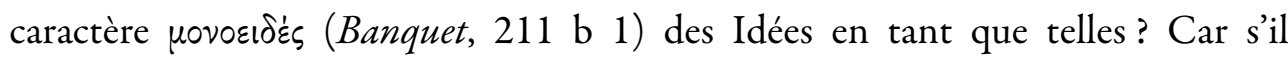
appartient à toute Idée d'avoir une forme et un contenu, cette dualité même doit constituer une marque distinctive de l'idéalité. D. semble oublier que ce par quoi les Idées se font remarquer auprès de l'intellect, c'est précisément ce qu'il appelle leur «contenu», justement parce qu'il implique des différences avec d'autres contenus possibles, en sorte que si ce contenu demeure extérieur à l'être-idée de l'Idée, alors l'Idée ne sera plus intégralement intelligible — son être-idée restera comme un point aveugle -, ce qui non seulement ira contre la lettre des 
Dialogues, mais en plus la rendra plus mythologique de jamais. À moins, bien sûr, que cette forme des Idées ne devienne à son tour intelligible en s'incarnant dans le contenu d'une Idée singulière. C'est, de fait, la voie que choisira D. en faisant du bien l'intelligibilité même de l'intelligible. Le bien sera donc en quelque sorte l'objectivation intuitionnable des conditions de possibilité transcendantales en se conformant auxquelles un contenu de pensée peut accéder au rang d'Idée. Mais si la forme peut ainsi passer ultimement du côté du contenu, cette distinction a-telle encore un sens fixe?

Maintenant que le rôle de la division quadripartite des êtres dans l'économie d'ensemble du Philèbe a été précisé, on peut montrer effectivement, par une application effective de la dialectique aux plaisirs et aux sciences, que la vie mixte bien hiérarchisée est un mélange de peras et d'apeiron. Ici, vu la nouveauté de l'objet dans l'histoire de la philosophie, la dialectique se fera procédé inventif plus que jamais, d'où des va-et-vient incessants entre les divers niveaux d'unité et de multiplicité. Et le rapport sous lequel le genre plaisir sera divisé concernera à chaque fois les modalités de son association aux diverses activités cognitives patronnées par l'intelligence. L'on verra alors que ce sont certaines modalités de cette association qui sont capables de rendre le plaisir bon, c'est-à-dire enfin vraiment désirable même pour un hédoniste, car le plaisir n'est pas bon par lui-même. Cette association impose du peras à l'apeiron natif du plaisir, apeiron que le plaisir partage avec la douleur. Découvrir quels sont les plaisirs bons, c'est donc aussi bien analyser la vie mixte dans son ensemble en tant qu'elle s'organise autour d'un primat de l'intelligence, c'est-à-dire la vie bonne. Par exemple, les plaisirs esthétiques ne peuvent survenir qu'à l'égard d'un objet complexe où une proportion mathématique repérée par l'intelligence est à l'œuvre. Contre Plotin, il n'y a pas de beauté du simple. C'est donc par une induction à partir de l'ensemble des plaisirs effectivement admissibles dans la vie bonne qu'on pourra déterminer rétrospectivement l'Idée du bien en tant que critère ayant présidé à la formation de cet ensemble. Ainsi les plaisirs faux recouvriront les plaisirs mélangés, tandis que seuls les plaisirs purs - un dénombrement exhaustif des espèces de plaisir oblige à reconnaître que tout plaisir n'est pas cessation progressive de la douleur - sont vrais et bons: l'intelligence gouverne intégralement les seconds. En revanche, toutes les sciences sont naturellement bonnes, et donc toutes pourront être admises dans la vie 
bonne. Du coup elles présentent un intérêt méthodologique moindre: leur division ne montre pas le critère du bien à l'œuvre.

Qu'en est-il donc de cette fameuse Idée du bien pour laquelle l'intellect a construit une « demeure », à savoir la vie bonne?

Le contenu de l'Idée du bien est censé se donner à nous intégralement dans la triade «mesure », « beauté », « vérité » (cf. Philèbe, 64d-e), en tant qu'on conçoit ces notions comme nécessairement solidaires. Ce sont là non pas simplement le «vestibule » (p. 583) du bien, comme si elles demeuraient dans une sorte d'extériorité à son égard, mais le visage du bien que l'on découvre une fois que l'on a franchi le vestibule - la description de la vie mixte par l'énumération de ses parties constituantes - qui mène à sa demeure. Le bien n'a donc plus rien d'ineffable. En lui s'atteste l'unité originaire de l'éthique, de l'esthétique et de l'épistémologie. La mesure est en effet la première propriété qui rend bon un mélange quelconque de peras et d'apeiron. Dans la mesure où elle peut s'appliquer à des réalités parfaitement mesurées en elles-mêmes et non mesurées par leur conformité à un étalon extérieur, la mesure peut s'appliquer à la pensée et est la condition de la vérité. L'erreur n'est jamais qu'indétermination. Mais de telles réalités intrinsèquement mesurées peuvent entrer en relation mutuelle au sein d'un tout organique, selon une juste proportion: on a affaire alors à la beauté. La beauté de la vie bonne résulte ainsi de l'association harmonieuse - et donc hiérarchisée - de ses divers moments, lesquels sont tous parfaitement mesurés en eux-mêmes. La proportion (ou commensurabilité) intervient elle aussi du côté de l'épistémologie car, si « la connaissance consiste avant tout à savoir reconnaître les identités et les différences entre cette chose et toutes les autres $\gg($ p. 581), il faut que l'objet de la connaissance s'articule au sein d'un tout sans pour autant se confondre avec les autres parties, bref il faut que cet objet soit beau, ainsi que les sont les Idées en général. Chacun des termes de la triade implique donc nécessairement les deux autres. Ils sont néanmoins ordonnés selon une certaine progression : la Mesure concerne chaque chose dans son rapport à elle-même, la Beauté dans son rapport au tout, et la Vérité dans son rapport à la connaissance.

Mais aussitôt surgissent plusieurs difficultés. Tout d'abord, si le bien n'est ce qu'il est que par le recoupement de ces trois caractères, n'est-ce pas par l'un au moins à titre de « cause logique » — que le bien est bien, en sorte que l'un est 
au dessus du bien dans la hiérarchie des principes? Ensuite, ou bien cette triade désigne trois effets du bien dans notre intellect, ou au moins sa «puissance » (64e) et alors ce n'est plus le bien lui-même en son essence - or la multiplicité des termes en question oblige à faire de ces termes quelque chose de relatif à l'intellect puisque le bien lui-même, en vertu de sa position de premier principe, est censé être parfaitement un - ou bien, si le bien est épuisé par cette triade, le bien n'est plus absolument premier. D., selon nous, ne parvient pas réconcilier l'exigence d'une connaissance immédiate de l'essence du bien lui-même et la multiplicité au moins nominale de ses déterminations qui s'impose dans le Philèbe. Il met en évidence les faiblesses de l'interprétation néoplatonicienne, notamment proclienne, sans fournir à la place une solution parfaitement satisfaisante. Mais peut-être s'agit-il ici d'une difficulté interne à la pensée de Platon.

Après avoir examiné l'Idée du bien en elle-même, il faut rendre compte de sa puissance à l'égard des autres Idées. L'Idée du bien a pour contenu singulier la forme commune à toutes les autres Idées et est cause de cette forme, de même que toute Idée est cause de ce que ses participants manifestent certaines ressemblances avec son contenu. Le bien, loin de constituer un genre commun à tous les contenus idéaux, est cause logique de l'idéalité que partagent toutes les Idées en tant que telles. C'est le bien qui les rend parfaitement déterminées en ellesmêmes. En cela le bien est cause de la vérité de la science car cette vérité ne consiste pas en l'adéquation de la pensée à une réalité extérieure, mais en la parfaite exactitude intrinsèque de notre pensée, laquelle ne peut survenir que lorsqu'on pense une Idée, quelle qu'elle soit, à partir du moment où toutes les Idées se caractérisent également elles-mêmes par leur parfaite détermination interne. Seule une telle exactitude de la pensée rend possible son adéquation avec le contenu singulier qu'elle pense.

De manière assez inattendue, $D$. refuse d'identifier le principe « anhypothétique » de la fin du livre VI de la République (511 b6) précisément à l'Idée du bien, préférant voir dans ce terme y une désignation de n'importe quelle Idée posée au début du processus dialectique, car une telle identification mènerait nécessairement, pense-t-il, à la doctrine proclienne de l'ineffabilité et de l'incognoscibilité du bien, ou au moins au fait qu'il ne peut pas être connu comme les autres idées, dans un rapport scientifique et objectif. Or une telle incognoscibilité se tient en contradiction avec les affirmations explicites et 
réitérées de Socrate (cf. $\mu \dot{\varepsilon} \gamma \mid \sigma \tau o \nu \mu \dot{\alpha} \theta \eta \mu \alpha$, République VI, 505a). En effet, si le bien est l'anhypothétique, il ne peut être connu, semble-t-il, à partir d'autre chose, et donc il ne peut être saisi qu'intuitivement et non par les opérations dialectiques de l'intellect, sous peine de régression à l'infini. Si D. rejette l'identification proclienne du bien à l'anhypothétique, c'est en réalité parce qu'il accepte son raisonnement.

Pourtant ce raisonnement proclien pèche par la confusion de la discursivité mathématique et de la discursivité dialectique, confusion que Socrate précisément dénonce à la fin du livre VI de la République. Connaître dialectiquement, ce n'est pas tirer des conclusions à partir de prémisses intangibles à la manière des démonstrations géométriques. La dialectique suppose un dialogue au moins de l'âme avec elle-même, obligeant à mettre au jour des couches toujours plus profondes parmi les souvenirs qui structurent l'intellect individuel, plutôt que l'application de procédures syllogistiques qui pourraient, à la limite, se passer de tout sujet et où la conclusion ne contient rien de plus de que les prémisses. L'exploration de la part nécessaire de notre intériorité ne se confond pas avec une production de conclusions toujours plus éloignées de la certitude initiale. La dialectique constitue, pour ainsi dire, un mouvement centripète, tandis que la discursivité mathématique est centrifuge. D. lui-même a bien montré que l'unité de l'Idée posée initialement dans les processus de division ne gagnait pour nous un contenu qu'au terme de ce processus, sans que cela produise pour autant une justification de cette Idée à partir d'autre chose qu'ellemême ; pourquoi faudrait-il donc qu'il en aille autrement pour l'Idée de ce qui est, en soi, anhypothétique ? Donc rien n'empêche qu'on connaisse dialectiquement l'anhypothétique.

D. argue en outre du fait que la saisie du « principe anhypothétique » est censée précéder, dans la République, la saisie des Idées, alors que le Bien constitue au contraire le terme et la fin de l'ascension dialectique à travers les Formes intelligibles. Mais ce serait pourtant là le moment de faire jouer sa distinction entre forme et contenu de l'intelligible : le mathématicien n'a pas besoin de la connaissance du Bien pour définir ses objets, poser ses axiomes et en déduire des théorèmes. Son discours peut se déployer sans l'intelligence proprement philosophique de ses objets. Ainsi, certes, son discours ne sera pas à proprement une science car l'âme n'y trouvera ni la certitude ni l'exactitude qui font la scientificité de la science; mais les théorèmes formulées pourront être tout à fait 
corrects, et ils pourront se multiplier indéfiniment selon une logique déductive. Ce qu'apporte la connaissance du principe anhypothétique, c'est bien plutôt la nécessité de donner le statut d'ov̉ซi à ces objets mathématiques, statut opposé au conventionnalisme qu'aurait pu revendiquer un Protagoras. Or, de l'avis même de D., c'est justement le Bien qui donne l'être essentiel, avec sa Mesure, sa Beauté et sa Vérité à tout le genre intelligible (cf. p. 591-592). Donc qu'il y ait un retour vers les objets mathématiques depuis le principe anhypothétique n'empêche pas que ce principe soit lui-même connu au terme d'une ascension dialectique de Forme en Forme, donc puisse s'identifier au Bien. L'ignorance où le dialecticien se trouve avant l'accès au principe anhypothétique n'est pas celle de la Justice ou de la Piété dans leur singularité. D., suivant encore une fois Proclus dont il voudrait se démarquer, force le texte de la République en y lisant que la connaissance de l'anhypothétique rendrait enfin possible «la saisie des Idées » (p. 8) en général, ou encore « la nature de chaque chose » (p. 7). Le texte insiste au contraire sur le fait que les objets qui deviendront intelligibles à la lumière du vrai «principe», à savoir l'anhypothétique, sont déjà les objets des « disciplines » subordonnées à la dialectique telles que la géométrie. Les Formes $(\varepsilon ` \delta \eta)$ dont il s'agit en $510 \mathrm{c} 1-2$ et auxquelles seules le dialecticien recourt dans sa redescente depuis l'anhypothétique ne sont pas des Formes encore jamais vues, mais les objets mathématiques désormais seulement révélées dans leur statut de Forme. Il est d'ailleurs étrange que D. ne mentionne pas à ce propos le texte bien connu du Phédon où, Socrate, usant déjà du vocabulaire de l'hypothèse, incite ses disciples à rechercher quelque chose de «suffisant » ( $\tau \iota$ ícavóv, 101 el) après avoir posé les premières hypothèses concernant l'intelligible, ce qui oblige à reconnaitre que ces premières hypothèses ne se confondent pas avec l'anhypothétique lequel, par définition, se suffit à lui-même puisqu'il ne dépend plus d'une autre hypothèse.

Enfin le bien, selon D., est également cause à l'égard du sensible, cause précisément qu'il soit toujours quelque chose, bien qu'il ne produise pas telle chose plutôt que telle autre en son contenu ni, par conséquent, ne soit responsable du passage de tel contenu à tel autre, bref du devenir. Or on ne peut être sans être quelque chose de déterminé. Donc le bien est la cause logique de tout ce qui est, tant dans l'intelligible que dans le sensible. La caverne de la République ne contenait-elle pas une ouverture par où filtrait la vraie lumière du jour? En revanche, alors que le bien est la cause de l'intelligibilité même de 
l'intelligible, étant lui-même, en sont contenu, cette intelligibilité sur un mode éminent, il n'est pas cause du sensible dans sa différence générale à l'égard de l'intelligible - car alors il serait cause du devenir - ni de l'articulation de ces deux plans de réalité, c'est-à-dire la participation. Seul un intellect démiurgique, pensant les Idées à titre de causes finales, est cause de la participation du sensible à l'intelligible en général et, par l'intermédiaire de ce dernier, cause de participation du sensible à la Mesure, à la Beauté et à la Vérité, car cette mise en participation est toujours une production. Mais une fois que cette participation est advenue, la bonté du sensible a pour seule cause le bien lui-même. Que dire alors d'un être sensible qui participe à une Idée parfaitement définie - quant à sa forme - de quelque chose de mauvais, tel un apeiron non mêlé de peras ? Car il y a de telles Idées ainsi que la division dialectique des plaisirs l'a révélé. Cet être sensible sera-til bon malgré tout ? Non. En réalité, le mal dans le sensible vient toujours de sa forme de sensible, alors que la participation à l'Idée décide du contenu de la chose sensible. L'assimilation du sensible et de l'intelligible ne peut se jouer que sur le plan du contenu de l'un et de l'autre, en sorte que la participation du sensible à l'intelligible ne peut s'effectuer que relativement à des Idées qui ont un contenu déterminé. Au contraire l'Idée d'apeiron, sous laquelle on peut regrouper certaines réalités sensibles, même si elle est parfaitement déterminée quant à sa forme, est en fait l'Idée d'un manque de participation entre le sensible et l'intelligible; elle ne saurait donc être cause d'une quelconque assimilation du sensible à l'intelligible en sa forme. Certaines choses sensibles ont beau se définir exclusivement par le caractère illimité, cela ne les rend pas limité pour autant. Il y a une sorte de chiasme: c'est seulement par son contenu que le sensible peut imiter la forme de l'intelligible; par conséquent seules certaines Idées peuvent ainsi le rehausser et le bonifier. Autrement dit, il y a bien des Idées de tout ce que peut vouloir connaître le dialecticien, même de la boue et de la crasse, ainsi que le disait Parménide au jeune Socrate, sans pour autant que la boue et la crasse soit une bonne chose. L'intellect démiurgique, ou l'intellect de l'homme politique véritable aura donc à sélectionner les Idées d'après lesquelles il veut produire le monde. Le critère de cette sélection sera évidemment le bien lui-même. D’où la nécessité de la connaître en ayant parcouru la totalité dialectique du Philèbe, même pour régler les affaires humaines. Et enfin chacun, au niveau de la petite cité qu'est son âme, devra aussi produire une vie bonne en se réglant sur le bien. L'Idée du bien n'est donc pas seulement ce qui permet de reconnaître le caractère

PLATO, The electronic Journal of the International Plato Society, n 10, 2010.

http://gramata.univ-paris1.fr/Plato/article96.html

(c) All rights of reproduction of any form reserved. 
commun à toutes les choses bonnes, mais est elle-même, en tant que réalité existant par soi, le plus grand des biens. Cela se traduira dans la hiérarchie finale du Philèbe: en premier lieu vient l'Idée du bien elle-même en tant qu'elle se diffracte en Mesure et Beauté, la Vérité étant impliquée par l'intelligence et la pensée qui viennent à la troisième place et ne s'exercent pleinement que dans la dialectique, puis viennent toutes les sciences et techniques particulières, et enfin les plaisirs purs de toute douleur relevant exclusivement de l'âme. Il n'y a pas de sixième rang: les plaisirs nécessaires qui accompagnent la vie bonne l'accompagnent parce qu'ils sont nécessairement liés à toute forme de vie et non parce qu'ils sont intrinsèquement bons.

Voilà quelques-uns des thèmes abordés dans cet ouvrage d'une richesse exceptionnelle. On peut ne pas partager les solutions apportées par D., mais on doit reconnaître qu'il repère les problèmes fondamentaux et les formule toujours avec acuité. Les objections que son interprétation du Philèbe peut susciter sont toujours l'expression d'une difficulté dans la pensée même de Platon. Ce livre est assurément destiné à devenir un ouvrage de référence parmi les études platoniciennes, tant par la rigueur de ses enchaînements conceptuels et son élan philosophique, en sympathie avec l'esprit originel du platonisme, que par l'ampleur de son érudition. 